\title{
Variables de Personalidad que motivan hacia el tratamiento
}

\author{
Francisca lópez-Torrecillas' ${ }^{1}$, Antonio Verdejo ${ }^{2}$, Marina J. Muñoz-Rivas ${ }^{3}$, Vicente Caballo ${ }^{2}$, José L. Graña ${ }^{4}$ \\ 1) Instituto de Neurociencias Dr. Oloriz; Departamento de Personalidad, Evaluación y Tratamiento Psicológico. \\ Facultad de Psicología. Universidad de Granada. \\ 2) Departamento de Personalidad, Evaluación y Tratamiento Psicológico. Facultad de Psicología. Universidad de Granada. \\ 3) Departmento de Psicología Biológica y de la Salud. Facultad de Psicología. Universidad Autónoma de Madrid. \\ 4) Departmento de de Personalidad, Evaluación y Tratamiento Psicológico. Facultad de Psicología. Universidad Complutense de Madrid.
}

Enviar correspondencia a:

Francisca López-Torrecillas. Instituto de Neurociencias Dr. Oloriz; Departamento de Personalidad, Evaluación y Tratamiento Psicológico. Facultad de Psicología. Universidad de Granada. Campus Universitario de Cartuja s/n. TLF: 958-249557. FAX: 958 243749. 18071 GRANADA. E-mail: fcalopez@.ugr.s

Recibido: 14 de junio de 2004 Aceptado: 6 de octubre de 2005.

\section{RESUMEN}

El objetivo del presente estudio fue analizar las diferencias existentes en autoeficacia, autocontrol, estilo atribucional y ansiedad social entre los drogodependientes que demandan tratamiento en comparación con aquellos que no lo hacen. El criterio de selección de los sujetos participantes fue la presencia de un trastorno por dependencia a drogas y/o alcohol según criterios del Manual Diagnóstico y Estadístico de los Trastornos Mentales (DSM-IV, DSM-IV-TR) (APA, 1994; 2000). Los sujetos fueron asignados a dos grupos en función de si demandaban o no tratamiento para su drogodependencia: Grupo clínico o de tratamiento $(n=50)$ y Grupo control o de no tratamiento $(n=34)$. Los resultados señalaron diferencias significativas siendo las puntuaciones del grupo de demanda de tratamiento más bajas en autocontrol y en autoeficacia social, más altas en ansiedad social y un estilo atribucional más interno y estable para los éxitos que el grupo de no demanda de tratamiento. Se analizan las implicaciones de los resultados para incrementar la demanda de tratamiento en adictos y su adhesión al mismo.

Palabras-clave: Personalidad, Demanda, Tratamiento.

\section{ABSTRACT}

The aim of this study was to analyse the differences in self-efficacy, self-control, attributional style, and social anxiety among drug dependents who request treatment compared with those who do not. The selection criterion for participants was the presence of a drug and/or alcohol dependence disorder according to the criteria of the Diagnostic and Statistical Manual of Mental Disorders (DSM-IV, DSM-IV-TR) (APA, 1994, 2000). Participants were assigned to two groups, clinical or treatment group ( $n=50$ ) and control or nontreatment group $(n=34)$. Results revealed significant differences between the groups, with lower scores in self-control and social self-efficacy, higher scores in social anxiety, and a more internal and stable attributional style for successes in the group that requested treatment compared with the group that did not request treatment. The implications of the results were analysed in order to increase the request for treatment by addicts and their adherence to it.

Keywords: Personality, Demand, Treatment.

\section{INTRODUCCIÓN.}

C uando se realizan balances sobre la utilidad de los servicios asistenciales para los drogodependientes, se hace notable que las tasas de sujetos que realizan tratamiento son inferiores al nivel estimado de drogodependientes existentes. Estos hallazgos han suscitado una reciente preocupación sobre aquellas variables que diferencian a los drogodependientes que demandan tratamiento de aquellos que no lo demandan.

Es bien conocido (Finney y Moss, 1995; Kline, 1996; Weisner, 1993) que las perturbaciones emocionales, los problemas de salud, los conflictos sociales e interpersonales y los problemas laborales inciden como presión para demandar tratamiento. Es más probable que sujetos con abuso y/o dependencia de alcohol, demanden tratamiento si perciben su problema como más severo, es decir, si han experimentado más síntomas de dependencia, mayor cantidad de consecuencias adversas relacionadas con el alcohol y/o drogas y más estresores en la mayoría de los ámbitos de su vida.

También se ha analizado (Marlowe et al., 1996) la influencia diferencial de las presiones coercitivas versus no coercitivas sobre la demanda de tratamiento. 
Tradicionalmente la coerción se ha definido en función del dictamen por parte de la justicia aunque, últimamente, se le está dando una mayor importancia a las presiones procedentes de la red social como variable coercitiva para la demanda de tratamiento. Aizpiri y Marcos (1996); Rodríguez-Martos et al. (1999) señalan que la familia, los amigos, los responsables laborales y los sistemas sanitario y legal "hacen ver" al alcohólico y/o drogodependiente la necesidad de analizar las consecuencias negativas del mantenimiento de su conducta adictiva y consecuentemente demandar tratamiento.

Las variables anteriormente mencionadas son esencialmente estáticas por lo que no son susceptibles de modificación, no están bajo el potencial control directo de los programas de intervención para facilitar el proceso de cambio del drogodependiente.

El modelo transteórico de Prochaska y DiClemente, (Prochaska y DiClemente, 1984; 1992; Prochaska, DiClemente y Noscross, 1992) conceptualiza la motivación para demandar tratamiento como un estado susceptible de cambio (Brownell et al., 1986; Saunders y Allsop, 1987; Van Bilsen y Van Emst, 1986) por lo que nos permite aproximarnos a factores dinámicos que si están bajo el control potencial de los programas de tratamiento y también nos permite integrar otras variables modificables mediante intervenciones terapéuticas, no originarias del modelo (Miller, 1985).

El modelo trasteórico de cambio de Prochaska y DiClemente, (Prochaska y DiClemente, 1984; 1992; Prochaska, DiClemente y Noscross, 1992) explica que la decisión de demandar tratamiento se basa en tres constructos: las etapas o estadios de cambio, el balance decisional y la autoeficacia. Existen varios pasos que precenden y siguen a la toma de decisión de una persona sobre si debe seguir consumiendo drogas o no; dichos pasos son descritos dentro de un proceso dinámico, que implica la progresión a través de cinco estadios de cambio: Precontemplación, contemplación, preparación, acción y mantenimiento.

Con respecto al estadio de precontemplación, cuando los drogodependientes están situados en este estadio aún no se han planteado modificar su conducta adictiva. La falta de reconocimiento del problema o déficit de "insight" se ha sido citado (Hingson et al., 1982; Kline, 1996; Longshore, Hsieh y Anglin, 1993; McLennan et al., 1998; Rounsaville y Kleber, 1985) como factor determinante de la resistencia a la búsqueda de tratamiento. Son muchos los sujetos a los que les cuesta autoidentificarse como individuos que tienen problemas con el alcohol y/o las drogas, es decir, no se evalúan a sí mismos como teniendo un problema. Si acuden a tratamiento lo hacen presionados por su entorno familiar, social o laboral.
La utilidad percibida del tratamiento por parte del drogodependiente, es una variable que habría que tener en cuenta en la etapa de precontemplación.; en esta línea, existen una serie de estudios (Fiorentine, Nakashima y Anglin, 1999; Kline, 1996; Longshore, Hsieh y Anglin, 1993) que resaltan que para que exista demanda de tratamiento los drogodependientes deben considerar la utilidad del mismo; encuentran, también, una relación directa y positiva entre expectativas positivas hacia el tratamiento (utilidad percibida del tratamiento) y demanda de tratamiento; en este sentido Rounsaville y Kleber (1985) señalan que los factores que influyen en la demanda de tratamiento son: la accesibilidad a los centros, la disponibilidad de servicios comunitarios y la disponibilidad económica; también se ha encontrado una asociación directa y positiva entre la demanda de tratamiento y las sesiones educativas por parte de trabajadores comunitarios (Booth, Crowley y Zhang, 1996).

Siguiendo con el modelo transteórico de cambio, en el estadio de contemplación los drogodependientes van tomando conciencia de su problema pero no han desarrollado aún un compromiso firme de cambio. Es en el estadio de preparación donde el drogodependiente toma la decisión y se compromete a abandonar su adicción. La etapa en la que el individuo cambia es denominada por el modelo como de acción, para posteriormente, pasar al mantenimiento de la abstinencia (estadio de mantenimiento). Si el mantenimiento de la abstinencia falla el drogodependiente pasa al estadio de recaída.

En el segundo constructo del modelo, en el balance decisional, se encuentran los procesos de cambio que se refieren a la forma en que los individuos van cambiando su conducta, incluyendo sus actuaciones manifiestas y encubiertas y se define como la valoración de las ventajas frente a las desventajas de usar las drogas. Desde la perspectiva del modelo transteórico del cambio, las personas que se sitúan en las primeras etapas de precontemplación y contemplación niegan las consecuencias negativas del consumo y se centran fundamentalmente en los efectos placenteros de las mismas. Según se avanza en las etapas, la tendencia se hace inversa, los efectos placenteros del consumo de drogas se valoran mucho menos y se le otorga mayor valor a las consecuencias negativas del consumo.

El tercer constructo del modelo es la autoeficacia, propuesta por Bandura (1982) e integrada dentro del modelo transteórico del cambio y definida como la convicción por parte del individuo de que es capaz de ejecutar exitosamente la conducta requerida para producir los resultados deseados. La autoeficacia percibida parece afectar a las decisiones del individuo para cambiar su conducta, al grado de esfuerzo dispuesto a invertir en el cambio y al mantenimiento a largo plazo 
de los cambios conductuales realizados. Sklar, Annis y Turner (1997) demostraron que altos niveles de autoeficacia estaban asociados a una menor dificultad percibida para abandonar las drogas, mayor motivación para abandonarlas y mayor confianza en la propia capacidad para dejarlas. Altos niveles de autoeficacia han sido asociados con demanda de tratamiento (Kessler et al., 1996).

En cuanto a los estadios de cambio, las subescalas de acción y mantenimiento están positivamente correlacionadas con altos niveles de autoeficacia (Miller y Rollnicks, 1991). Diclemente y Hughes 1990) afirman que la autoeficacia se relaciona con la voluntad para cambiar de conducta; en su estudio evalúan la autoeficacia para la abstinencia en el contexto de los estadios del modelo de cambio con sujetos alcohólicos que estaban dentro de un programa de tratamiento externo. Se observó que tanto la autoeficacia para la abstinencia como las tentaciones de beber estaban relacionadas con el estadio de cambio de cada sujeto; entre aquellos que estaban en un estadio de precontemplación o contemplación, tenían el nivel más bajo de autoeficacia y los niveles más altos de tentaciones. En la misma línea, Rounsaville et al. (1991) señalaban que entre las manifestaciones que presentaban los sujetos que demandaban tratamiento aparecían la falta de confianza para abstenerse, baja autoeficacia, percepción deficitaria de apoyo social y déficits en el funcionamiento social. Bajos niveles de autoeficacia estaban relacionados con la ausencia de control interno sobre el uso de sustancias adictivas y con subescalas de situaciones negativas relacionadas con emociones desagradables, conflicto interpersonal y-para los cocainómanos- disconfort físico.

El modelo transteórico de Prochaska y DiClemente, (Prochaska y DiClemente, 1984; 1992; Prochaska, DiClemente y Noscross, 1992) conceptualiza la motivación para demandar tratamiento como un estado suceptible de cambio (Brownell et al., 1986; Saunders y Allsop, 1987; Van Bilsen y Van Emst, 1986) por lo que nos permite aproximarnos a factores dinámicos que si están bajo el control potencial de los programas de tratamiento y también nos permite integrar otras variables modificables mediante intervenciones terapéuticas, no originarias del modelo (Miller, 1985). Proponemos integrar en el modelo variables de personalidad tales como: autoeficacia, estilo atribucional, autocontrol y ansiedad social. Dichas variables nos permitirían aproximarnos a factores dinámicos dado que están bajo el control del sujeto, por lo que podríamos diseñar estrategias de intervención que incidieran en la motivación para demandar tratamiento.

Aunque no existen estudios que relacionen el estilo atribucional con la demanda de tratamiento destacamos, por su interés, algunos hallazgos encontrados en el área de las drogodependencias. Parece que
(Dowd, Lawson y Petosa ,1986; Echeburúa y Elizondo, 1988; Reich y Gutiérres, 1987; Sterling et al., 1996) los drogodependientes presentan un estilo de pensamiento contrario a percepciones favorables (interno y estable para los éxitos), llegando a proponerse, incluso, como un predictor para el pronóstico del tratamiento. Nos parece importante considerar la pauta atribucinal contingente al éxito y/o al fracaso porque las acciones que persiguen fines específicos están mediadas por las atribuciones causales que constituyen, a su vez, un aspecto importante en las creencias de control; la realización de la acción requiere tanto la autobservación que resulta de la acción como el reconocimiento de que las acciones son parte de uno mismo; las atribuciones median los efectos de la autoeficacia sobre la ejecución futura y el éxito se logra normalmente mediante esfuerzos renovados tras los intentos fallidos; existe una relación bidireccional entre las atribuciones causales y las creencias de eficacia personal (Bandura, 1995).

El constructo de autoeficacia versa sobre las creencias de control y por tanto, indirectamente, sobre la ejecución del control (Diclemente, Fairhurst y Piotrowski, 1995). Desde la perspectiva de Rosembaum (1980). El autocontrol entendido como autobservación, autoevaluación y autorrefuerzo, se asocia con las etapas preparación, acción y mantenimiento del modelo transteórico de cambio. Si bien no existen estudios que relacionen directamente el autocontrol con la demanda de tratamiento, nos resulta interesante considerarlo, dado que, se encuentra (Annis y Davis, 1988; Tarter, Jacob y Bremen, 1989) que los drogodependientes no disponen de repertorios adecuados de habilidades de autocontrol, planificación y toma de decisiones.

Una última variable que sugerimos como influyente en la toma de decisión de demandar tratamiento es la ansiedad social. Pensamos que esta variable podría estar relacionada con la demanda de tratamiento porque Grenyer et al. (1992) señaló que los adictos a opiáceos presentaban altos niveles de ansiedad en el inicio del tratamiento para su dependencia. Aunque actualmente no exista evidencia alguna para enmarcar está variable dentro del modelo transteórico de cambio, puesto que el modelo permite integrar nuevas variables, plantemos la necesidad de analizarla.

Por tanto, podemos resumir resaltando la importancia de estudiar las variables de personalidad tales como autoeficacia, estilo atribucional, autocontrol y ansiedad social porque son susceptibles de cambio y podrían incidir en la motivación para demandar tratamiento, produciendo, por tanto, una mejora global en el proceso de tratamiento del alcohólico y/o drogodependiente.

El objetivo del presente estudio es analizar las diferencias de la autoeficacia, autocontrol, estilo atribucio- 
nal y ansiedad social en la demanda versus no demanda de tratamiento de los drogodependientes.

\section{METODOLOGÍA}

\subsection{SUJETOS}

Participaron en este estudio 84 sujetos (21 mujeres y 63 hombres). La selección de los sujetos se realizó en Centros de Drogodependencias y en lugares de encuentro de jóvenes de Andalucía, todos ellos completaron de forma voluntaria la entrevista, cuestionarios y escala que a continuación se describen. El criterio de selección fue que los sujetos tuvieran un trastorno por dependencia a drogas y/o alcohol según criterios del DSM-IV y DSM-IV-TR (APA, 1994; 2000).

Los sujetos fueron asignados a los siguientes grupos, según demandaran tratamiento para su drogodependencia o no:

- Grupo de demanda de tratamiento (clínico): (n = 50) compuesto por 38 hombres y 12 mujeres, con una edad media de 26.12 años y desviación típica de 4.59. Fueron seleccionados en Centros de Drogodependen- cias de Andalucía cuando iban a demandar tratamiento para su drogodependencia; mediante la Entrevista de Investigación acerca del Comportamiento Adictivo (E.I.C.A) detectabamos que consideraban tener serios problemas con las drogas por lo que esta era una razón importante para demandar tratamiento.

- Grupo control o de no tratamiento: $(n=34)$ compuesto por 25 hombres y 9 mujeres, con una edad media de 26.74 años y desviación típica de 4.76. Fueron seleccionados en Centros de Drogodependencias de Andalucía cuando iban a acompañar a un familiar o amigo para demandar tratamiento para su drogodependencia; también fue seleccionado en lugares de encuentros de jóvenes de Andalucia; mediante la Entrevista de Investigación acerca del Comportamiento Adictivo (E.I.C.A) detectabamos que no consideraban tener problemas con las drogas por lo que no consideraban la necesidad de realizar tratamiento.

En la adscripción de los sujetos a los grupos se controló la edad (t $(82)=-0.59 ; p=0.554)$, genero $(\chi 2(1)$ $=0.066 ; \mathrm{p}=0.797)$, nivel cultural $(\chi 2(5)=2.21 ; \mathrm{p}$ $=0.820)$ y categoría profesional $(\chi 2(10)=6.49 ; \mathrm{p}=$ 0.773) (ver tablas 1, 2 y 3).

Para comprobar si la severidad del consumo de alcohol y drogas (cantidad y cronicidad del consumo de alcohol, hachís, cocaína y heroína) diferenciaba a

Tabla 1. Media y desviación típica de la edad y distribución de género de los grupos

\begin{tabular}{|l|cc|cc|}
\hline \multirow{2}{*}{ GRUPOS } & \multicolumn{2}{|c|}{ EDAD } & \multicolumn{2}{c|}{ GÉNERO } \\
\cline { 2 - 5 } & Media & Desv. típica & Hombres & Mujeres \\
\hline DEMANDA & 26.12 & 4.59 & 38 & 12 \\
NO DEMANDA & 26.74 & 4.76 & 25 & 9 \\
\hline
\end{tabular}

Tabla 2. Nivel educativo de los grupos

\begin{tabular}{|l|cccccc|}
\hline GRUPOS & \multicolumn{5}{|c|}{ NIVEL EDUCATIVO } \\
\hline ESTUDIOS & PRIMARIOS & SECUNDARIOS & EN DIPLOMATURA & DIPLOMADOS & EN LICENCIATURA & LICENCIADOS \\
\hline DEMANDA & 34 & 10 & 1 & 2 & 2 & 1 \\
NO DEMANDA & 18 & 9 & 1 & 2 & 3 & 1 \\
\hline
\end{tabular}

\begin{tabular}{|c|c|c|c|c|c|c|c|c|c|c|c|}
\hline \multicolumn{12}{|c|}{ Tabla 3. Situación laboral de los grupos } \\
\hline \multirow[b]{2}{*}{ GRUPOS } & \multicolumn{10}{|c|}{ SITUACIÓN LABORAL } & \multirow[b]{2}{*}{ Servicios } \\
\hline & $\begin{array}{c}\text { No } \\
\text { trabaja }\end{array}$ & Técnicos & $\begin{array}{l}\text { Empresas } \\
\text { públicas }\end{array}$ & Comercio & Hotelería & $\begin{array}{c}\text { Agricultura } \\
\text { y ganad. }\end{array}$ & Construc. & $\begin{array}{c}\text { Trab. no } \\
\text { especial. }\end{array}$ & $\begin{array}{c}\text { Indust. } \\
\text { manufac. }\end{array}$ & Construc. & \\
\hline DEMANDA & 24 & 1 & 1 & 2 & 1 & 8 & 5 & 1 & 1 & 3 & 3 \\
\hline NO DEMANDA & 18 & 1 & 1 & 3 & 1 & 2 & 3 & 0 & 1 & 0 & 4 \\
\hline
\end{tabular}


los grupos de demanda de tratamiento versus de no demanda de tratamiento, se realizó un Análisis Multivariado de la Varianza (MANOVA), para un diseño unifactorial entregrupos, utilizando la variables grupo (demanda de tratamiento versus no demanda de tratamiento) como factor y las puntuaciones estandarizadas relacionadas con el consumo de drogas ((1) puntuación típica de la cantidad de consumo de drogas, (2) puntuación típica de la cronicidad del consumo de drogas y (3) puntuación típica total -cantidad y cronicidad de consumo de drogas-) como variables dependientes.
Los resultados indicaron diferencias en el factor grupo (Wilks' Lambda $=0.861, F_{2,81}=6.56 ; p=0.002$ ). Los ANOVAs mostraron que había diferencias estadísticamente significativas del factor grupo en la variable cronicidad en el consumo de drogas $\left(F_{1,82}=12.51\right.$; Mce $=$ $3.48 ; p=0.001$ ) y en la variable de consumo total (cantidad y cronicidad del consumo de drogas) $\left(F_{1,82}=5.02\right.$; Mce $=1.30 ; p=0.028$ ). No se encontraron diferencias estadísticamente significativas en la cantidad de consumo de drogas $\left(F_{1,82}=0.372 ;\right.$ Mce $\left.=0.175 ; p=0.544\right)$ (ver tabla 4).

Tabla 4. Medias, desviaciones típicas y significatividad de las puntuaciones típicas de consumo de drogas en los grupos de demanda versus no demanda

\begin{tabular}{|l|cc|cc|c|}
\hline & \multicolumn{2}{|c|}{ GRUPO DEMANDA } & \multicolumn{2}{c|}{ GRUPO NO DEMANDA } & \multirow{2}{*}{$\boldsymbol{F}$} \\
\cline { 2 - 6 } & MEDIA & DESV. TIPICA & MEDIA & DESV. TIPICA & \\
\cline { 2 - 6 } CANTIDAD & 0.17 & 0.69 & 0.08 & 0.69 & 0.372 \\
CRONICIDAD & 0.18 & 0.58 & -0.24 & 0.44 & $12.51^{* * *}$ \\
TOTAL CONSUMO & 0.18 & 0.52 & -0.08 & 0.49 & $5.02^{*}$ \\
\hline $\mathrm{p}<0.001=; p<0.05=$
\end{tabular}

\subsection{MEDIDAS}

Las medidas utilizas fueron:

- Escala de Autoeficacia (A.E) de Sherer, Maddux, Mercandante, Prentice-Dunn, Jacobs y Rogers (1982).

- Cuestionario de Estilo Atribucional de Peterson et al. (1982) (A.S.Q).

- Cuestionario de Autocontrol (AC) de Rosenbaum (1980).

- Entrevista de Investigación acerca del Comportamiento Adictivo (E.I.C.A). Obtiene 3 puntuaciones estandarizadas (tipificadas) que definen el consumo de drogas, una es la cantidad de consumo de drogas, otra es la cronicidad de dicho consumo y la tercera es la suma de las dos anteriores que determina el grado de severidad de consumo de alcohol y/o drogas.

\section{RESULTADOS}

Para llevar a cabo el objetivo de nuestro estudio, procedimos a analizar si las variables relacionadas con la personalidad (autoeficacia -general, social y global-, autocontrol, estilo atribucional para los éxitos -interno versus externo, estable versus inestable, global versus específico-, estilo atribucional para los fracasos -interno versus externo, estable versus inestable, global versus específico- y ansiedad social -) diferenciaban a los grupos de demanda de tratamiento versus de nodemanda de tratamiento. Para ello, se realizó un Análisis Multivariado de la Covarianza (MANCOVA), para un diseño unifactorial entregrupos, utilizando la variables grupo (demanda de tratamiento versus no-demanda de tratamiento) como factor y las variables relacionadas con la personalidad como variables dependientes y como covariado la puntuación típica total de la cantidad y cronicidad de consumo de drogas.

Los resultados indicaron diferencias significativas del factor grupo (Wilks' Lambda $=0.585, F_{10,72}=5.10$; $p=0.000)$. No se encontraron diferencias significativas del covariado

$$
\text { (Wilks' Lambda }=0.830, F_{10,72}=1.472 ; p=0.168 \text { ) }
$$

Puesto que el MANCOVA mostró resultados estadísticamente significativos en el efecto grupo y no para el covariado (puntuación típica total de la cantidad y cronicidad del consumo de drogas), se procedió a realizar ANOVAs univariados para cada variable dependiente utilizando el factor grupo como variable independiente. Dichos ANOVAs mostraron que había diferencias estadísticamente significativas en autoeficacia social $\left(F_{1,81}=5.862 ;\right.$ Mce $\left.=107.132 ; p=0.018\right)$, en autocontrol $\left(F_{1,81}=4.694 ;\right.$ Mce $\left.=2984.667 ; p=0.033\right)$, en estilo atribucional interno versus externo para los éxitos $\left(F_{1,81}=11.145 ;\right.$ Mce $\left.=326.233 ; p=0.001\right)$, en estilo atribucional estable versus inestable para los éxitos 
Tabla 5. Resultados obtenidos para el grupo de demanda de tratamiento versus no demanda de tratamiento.

\begin{tabular}{|l|cc|cc|c|}
\hline \multirow{2}{*}{ VARIABLES } & \multicolumn{2}{|c|}{ GRUPO DEMANDA } & \multicolumn{2}{c|}{ GRUPO NO DEMANDA } & \multirow{2}{*}{$\boldsymbol{F}$} \\
\cline { 2 - 5 } & MEDIA & DESV. TIPICA & MEDIA & DESV. TIPICA & \\
\hline AE-1 & 60.56 & 11.09 & 62.71 & 7.51 & 1.040 \\
AE-2 & 20.46 & 4.44 & 23.03 & 4.00 & $5.862^{* *}$ \\
AE-3 & 81.02 & 14.32 & 85.74 & 9.55 & 2.583 \\
ASQ-E1 & 34.33 & 5.28 & 30.49 & 5.59 & $11.145^{* * *}$ \\
ASQ-E2 & 32.9 & 7.07 & 28.80 & 7.17 & $3.956^{*}$ \\
ASQ-E3 & 28.48 & 8.84 & 26.44 & 8.37 & 0.940 \\
ASQ-F1 & 21.83 & 7.42 & 23.68 & 7.11 & 0.488 \\
ASQ-F2 & 23.42 & 9.37 & 24.69 & 8.67 & 0.589 \\
ASO-F3 & 23.11 & 8.19 & 23.22 & 9.35 & 0.004 \\
AC & 16.32 & 26.61 & 31.21 & 23.79 & $4.69^{*}$ \\
FNES-B & 37.48 & 7.87 & 28.44 & 6.70 & $34.069^{* * *}$ \\
\hline
\end{tabular}

$p<0.001=; p<0.05=; p=0.05=$

$\mathrm{AE}-1=$ Autoeficacia general.

$\mathrm{AE}-2=$ Autoeficacia social.

$A E-3=$ Autoeficacia total.

ASQ-E1 = Estilo atribucional interno versus externo para los éxitos.

ASQ-E2 = Estilo atribucional estable versus inestable para los éxitos.

ASQ-E3= Estilo atribucional global versus específico para los éxitos.

ASQ-F1 = Estilo atribucional interno versus externo para los fracasos ASQ-F2 = Estilo atribucional interno versus externo para los fracasos ASQ-F3= Estilo atribucional interno versus externo para los fracasos. $\mathrm{AC}=$ Autocontrol.

FNES-B $=$ Ansiedad social.

$\left(F_{1,81}=3.956 ;\right.$ Mce $\left.=202.265 ; p=0.050\right)$ y en ansiedad social $\left(F_{1,81}=34.069 ;\right.$ Mce $\left.=1823.646 ; p=0.000\right)$, siendo las puntuaciones del grupo de demanda de tratamiento más bajas en autocontrol, en autoeficacia social y más altas en ansiedad social que las del grupo de no demanda de tratamiento. Además el grupo que demanda tratamiento presenta un estilo atribucional más interno, estable para los éxitos que el grupo de no demanda de tratamiento (ver tabla 5).

No se encontraron diferencias estadísticamente significativas en autoeficacia general y en la puntuación total de autoeficacia. Tampoco se encontraron diferencias en el estilo atribucional global versus específico para los éxitos, ni en el estilo atribucional interno versus externo, estable versus inestable y global versus específico para los fracasos.

\section{DISCUSIÓNY CONCLUSIONES.}

Los resultados indicaron diferencias significativas del grupo en la cronicidad de consumo de drogas y en la variable consumo total (cantidad y cronicidad del consumo de drogas) Por ello, realizamos un Análisis Multivariado de la Covarianza (MANCOVA), para un diseño unifactorial entregrupos. Aunque los resultados indicaron diferencias significativas del factor grupo, no se encontraron diferencias significativas para el cova- riado. Por lo que se demuestra que la severidad del consumo de alcohol y/o drogas no afecta a nuestras variables de estudio.

Los resultados mostraron diferencias en autoeficacia social, autocontrol, ansiedad social y estilo atribucional interno versus externo y estable versus inestable para los éxitos, siendo las puntuaciones del grupo de demanda de tratamiento más bajas en autocontrol, en autoeficacia social y más altas en ansiedad social que las del grupo de no demanda de tratamiento. Además, el grupo que demanda tratamiento presenta un estilo atribucional más interno y estable para los éxitos que el grupo de no demanda de tratamiento.

No se encontraron diferencias estadísticamente significativas en autoeficacia general y en la puntuación total de autoeficacia. Tampoco encontramos diferencias en el estilo atribucional global versus específico para los éxitos ni en el estilo atribucional interno versus externo, estable versus inestable y global versus específico para los fracasos.

Los resultados obtenidos en este estudio señalan diferencias en ansiedad social, siendo las puntuaciones del grupo de demanda de tratamiento más altas en ansiedad social que las del grupo de no demanda de tratamiento. Estos resultados son congruentes con los obtenidos por Grenyer et al. (1992), aunque, como mencionamos anteriormente, este estudio se realizó con drogodependientes en tratamiento. Si recordamos que Leary (1983) entiende la ansiedad social determinada por la presencia de una evaluación interperso- 
nal en lugares sociales, ya sean reales o imaginarios, cabe pensar, en el caso de los drogodependientes que realicen evaluaciones reales. Las consecuencias que conlleva el consumo prolongado de drogas en nuestra sociedad, son las críticas que se reciben. Por tanto, los individuos que deciden someterse a tratamiento, pueden estar sometidos a presiones (en forma de críticas) de su entorno interpersonal. Una de las estrategias de afrontamiento, pudiera ser someterse a tratamiento a fin de reducir esa ansiedad desencadenada por su entorno social. También podemos interpretar los resultados teniendo en cuenta la influencia diferencial de las presiones coercitivas, Marlowe et al. (1996) señalan como influyentes la coerción ó evitación mediada socialmente y la aprobación ó reforzamiento positivo mediado socialmente.

Otro efecto significativo, lo encontramos en la autoeficacia, concretamente en su dimensión social, es decir, los resultados mostraron diferencias en autoeficacia social, siendo las puntuaciones del grupo de demanda de tratamiento más bajas que las del grupo de no demanda de tratamiento. No se encontraron diferencias estadísticamente significativas en autoeficacia general y en la puntuación total de autoeficacia aunque si aparecen diferencias clínicas siendo la autoeficacia general y la puntuación total de la autoeficacia bajas para el grupo que demanda tratamiento.

Como señala Bandura (1995) el éxito se logra normalmente mediante esfuerzos renovados tras los intentos fallidos. Los logros humanos necesitan un sentido resistente de eficacia personal; el éxito requiere un esfuerzo perseverante, de manera que el sentido de la eficacia de las personas no se vea debilitado ante los primeros meses. Aunque nuestros resultados no son congruentes con los obtenidos por Kessler et al., (1996); Sklar, Annis y Turner (1997) quienes afirman que la autoeficacia se relaciona con la voluntad para cambiar de conducta, no podemos rechazar que la autoeficacia constituya un factor importante en el análisis de la demanda de tratamiento. Puesto que la autoeficacia es una variable dinámica cabría la posibilidad de que fluctuase a lo largo del proceso de tratamiento, esta afirmación quedaría apoyada por Diclemente y Hughes (1990), quienes observaron que la autoeficacia estaba relacionada con el estadio de cambio de cada sujeto; los sujetos que estaban en un estadio de precontemplación o contemplación tenían el nivel más bajo de autoeficacia que los que estaban en el estadio de acción, y Rounsaville et al. (1991), quienes señalaron que los sujetos que demandaban tratamiento presentaban falta de confianza para abstenerse y baja autoeficacia.

Los resultados mostraron también diferencias en autocontrol, esto es, el grupo que demanda tratamiento presenta puntuaciones más bajas que el grupo que no demanda tratamiento.
Cuando los sujetos se consideran con menor autocontrol demandan tratamiento en comparación con los que no lo demandan. Estos resultados se podrían explicar por el sentimiento de incapacidad de enfrentarse y controlar el consumo y la situación generada por este, derivando por tanto en la petición de ayuda externa mediante la demanda de tratamiento.

Finalmente, con respecto al estilo atribucional, encontramos que el grupo que demanda tratamiento presenta un estilo atribucional interno versus externo y estable versus inestable para los éxitos. Aunque no se encontraron diferencias estadísticamente significativas en el estilo atribucional global versus específico para los éxitos ni en el estilo atribucional interno versus externo, estable versus inestable y global versus específico para los fracasos observamos que aparecen diferencias clínicas. Parecer ser que los drogodependientes que demandan tratamiento presentan un estilo atribucional considerado por los autores (Dowd, Lawson y Petosa ,1986; Echeburúa y Elizondo, 1988; Reich y Gutiérres, 1987; Sterling et al., 1996) como más adaptativo o favorable.

Los resultados del estudio irían en la línea de la conceptualización del cambio defendida por Prochaska y Diclemente (1992), que proponen un modelo de fases de cambio para describir, explicar y predecir el proceso de abandono de diferentes conductas adictivas. En futuras investigaciones los estudios podrían dirigirse a determinar la relación de las variables de personalidad, que resultaron significativas en este estudio, con la fase de acción del modelo transteórico de Prochaska y Diclemente, entendiendo esta fase como la etapa en la que el individuo cambia, con o sin ayuda profesional, su conducta manifiesta y encubierta, así como las condiciones ambientales que le afectan, con el objetivo de superar la adicción.

Por tanto, las variables analizadas (ansiedad social, autoeficacia, autocontrol, estilo atribucional y ansiedad social) quedarían integradas de la siguiente manera: El constructo de autoeficacia, propuesto por Bandura (1982), se integra dentro del modelo transteórico del cambio, altos niveles de autoeficacia se asocian con los estadios de acción y mantenimiento (Miller, 1985) y bajos niveles de autoeficacia se relacionan con los estadios de precontemplación y contemplación (Diclemente y Hughes 1990; Rounsaville et al.,1991). Por otro lado, también conocemos la relación bidereccional entre la autoeficacia y las atribuciones causales (Bandura, 1995) y entre las creencias de control y la ejecución del mismo (Diclemente, Fairhurst y Piotrowski, 1995). Finalmente, con respecto a la ansiedad social, actualmente no existe evidencia alguna para enmarcar está variable dentro del modelo transteórico de cambio, sin embargo, puesto que el modelo permite integrar nuevas variables, plantemos la necesidad de incluirla. 


\section{REFERENCIAS}

American Psychiatric Association (1994). Diagnostic and statistical manual of mental disorders ( $4^{a}$ edición DSMIV). Washington, DC: Autor.

American Psychiatric Association (2000). Diagnostic and statistical manual of mental disorders ( $4^{a}$ edición- texto revisado DSM-IV-TR). Washington, DC: Autor.

Aizpiri Díaz J. y Marcos Frías J. F. (1996). Actualización del tratamiento del alcoholismo. Barcelona. Masson.

Annis, H. M. y Davis, C. S. (1988). Self-efficacy and the prevention of alcoholic relapse: Initial findings from a treatment trial. InT. B. Baker y D. Cannon (Eds.). Addictive Disorders: Psychological Research on Assesment and Treatment (pp 88-112). New York. Praeger.

Bandura, A. (1982). Self-efficacy mechanism in human agency. American Phychologist, 37, 122-147.

Bandura, A. (1995). Self-efficacy in changing societies. Cambridge: University Press.

Booth, R.E., Crowley, T.J. y Zhang, Y. (1996). Substance abuse treatment entry, retention and effectiveness out-of-treatment opiate injection drug users. Drug and Alcohol Dependence, 42, 11-20.

Brownell, K.D; Marlatt, G.A; Lichtenstein, E y Wilson, G.T (1986). The effects of repetead venting relapse. American Psychologist, 41, 765-782.

Diclemente, C.C. y Hughes, S.O. (1990). Stages of change profiles in outpatient alcoholism treatment. Journal of Substance Abuse, 2, 217-235.

Diclemente, C.C., Fairhurst, S.K. y Piotrowski, N.A. (1995). Self-efficacy and addictive behaviors. En J:E: Maddux (Ed.). Self-efficacy, adaptation, and adjustement: Theory, research, and application. New York: Plenum Press.

Down, E.T., Lawson, G.W. y Petosa, R. (1986). Attributional styles of alcoholics. The International Journal of the Addictions, 21, 589-593.

Echeburúa, E. y Elizondo, M. (1988). Estilo atribucional y locus de control en una muestra de alcohólicos anónimos. Revista Vasca de Psicologia, 1, 71-78.

Finney, J. W. y Moss, R. H. (1995) Entering treatment for alcohol abuse: a stress and coping model. Addiction, 90, 1223-1240.

Fiorentine, R., Nakashima, J. y Anglin, M. D. (1999) Client engagement in drug treatment. Journal of Substance Abuse Treatment, 17, 199-206.

Grenyer, B.F.S., Williams, G., Swift, W. y Neill, O. (1992). The prevalence of social-evaluative anxiety in opioid users seeking treatment. The International Journal of the Addictions, 27, 665-673.

Hingson, R., Magione, T., Meyers, A. y Scotch, N. (1982) Seeking help for drinking problems: A study in the boston metropolitan area. Journal of Studies of Alcohol, 43, 273-288.

Kessler, R.C., Nelson, C.B., McGonagle, K.A., Edlund, M.J., Frank, R.G. y Leaf, P. J. (1996). The epidemiology of cooccurring addictive and mental disorders: Implications for prevention and service utilization. American Journal of Orthopsychiatry, 66, 17-31.

Kline, A (1996). Pathways into drug user treatment: the influence of gender and racial/ethnic identify. Substance Use Misuse, 31, 323-342.

Leary, M.R. (1983). A brief versión of the fear of negative evaluation scale. Personality and Social Psychology Bulletin, 9, 371-376.

Longshore, D., Hsieh, S y Anglin, M.D (1993). Ethnic and gender differences in drug users' perceived need for treatment. International Journal of Addiction, 28, 539558.

Marlowe, D.B., Kirby, K.C., Bonieskie, L.M., Glass, D.J., Dodds, L.D., Husband, L.D., Platt, J.J. y Festinger, D.S. (1996) Assesment of coercitive and noncoercitive pressures to enter drug abuse treatment. Drug and Alcohol Dependence, 42, 77-84.

McLennan, J. D., Shaw, E., Shema, S. J., Gardner, W. P., Pope, S. K. y Kelleher, K. J. (1998) Adolescents'insight in heavy drinking. Journal of Adolescent Health, 22, 409-416.

Miller, W.R (1985). Motivation for treatment: a review with special emphasis on alcoholism. Psychological Bulletin, 98, 84-107.

Miller, W.R y Rollnicks, S. (1991). Motivational interviewing: preparing people to change addictive behavior. Nueva York: Guilford Press.

Peterson, C., Semmel, A., Von Baeyer, C., Abramson, L.Y., Metalsky, L.I., y Seligman, M.E.P. (1982). The attributional style questionnaiere. Cognitive Theraphy Research, 6, 287-300.

Prochaska, J.O y DiClemente, C.C (1984). The transtheorical approach: Crossing the traditional boundaries of therapy, Homewood, Illinois, Dorsey Press.

Prochaska, J.O y DiClemente, C.C (1992). Stages of change in the modification of problem behaviors. En: M, Hersen, R.M, Eisler y P.M, Miller (Eds). Progress in behavior modification, Newbury Park, California, Sage.

Prochaska, J.O, DiClemente, C.C y Norcross, J.C. (1992). In search of how people change. Applications to addictive behaviors. American Psychologist, 47, 1102-1114.

Reich, J.W. y Gutiérres, S.E. (1987). Life event and treatment attributions in drug abuse and rehabilitation. American Journal Drug Alcohol Abuse, 13, 73-94.

Rodríguez-Martos A., Altisent R., Aubá J., Freixa F., Gual A., Rodes J., y Santodomingo J. (1999). Curso de Formación sobre Prevención y Tratamiento del Alcoholismo. Barcelona Ediciones Doyma, S. A. 
Rosenbaum, M. (1980). Cuestionario de autocontrol. In A. Capafóns y P. Barreto (1989) (traducción y adaptación). Competencia aprendida: Fiabilidad y validez de su medida, críticas y recomendaciones. Revista Española de Terapia del Comportamiento, 1, 19-39.

Rounsaville, B.T., Anton, S.F., Carroll, K.M., Budde, M., Prosoff, B.A. y Gannin F.T. (1991). Psychiatric diagnosis of treatment seeking cocaine abusers. Archives of General Psychiatry, 48, 43-51.

Rounsaville, B.J. y Kleber H.D. (1985). Untreated opiate addicts: How do they differ from tose seeking treatment. Archives of General Psychiatry, 42, 1072-1080.

Saunders, B y Allsop, S. (1987). Relapse: A psychological view. British Journal of Addiction, 82, 417-429.

Sherer, M., Maddux, J.E., Mercandante, B., Prentice-Dunn, S., Jacobs, B. y Rogers, R.W. (1982). The Self-efficacy Scale: Construction and validation. Psychological Reports. 51, 663-671.
Sklar, S. M., Annis, H.M. y Turner, N.E. (1997). Development and validation of the drug-taking confidence questionnaire: a measure of coping self-efficacy. Addictive Behaviors, 22, 655-670.

Sterling, R.C., Gotteheil, E., Weinstein, S.P., Lundy, A. y Serota, R.D. (1996). Learned helplessness and cocaine dependence: An investigation. Journal of Addictive Diseases, 15, 13-24.

Tarter, R. E., Jacob, T. y Bremer, D. A. (1989). Cognitive status of sons of alcoholics. Alcoholism: Clinical and Experimental Research, 13, 232-235.

Van Bilsen, H.P.J.G y Van Emst, A.J. (1986). Heroin addiction and motivational milieu therapy. The International Journal of the Addictions, 21, 707-713.

Weisner, C. (1993). Toward an alcohol treatment entry model: a comparison of problem drinkers in the general population and in treatment. Alcohol: Clinical and Experimental Research, 17, 746-752. 
